\title{
Litter impacts on scenery and tourism on the Colombian north Caribbean coast
}

Williams, Allan Thomas; Rangel Buitrago, Nelson Guillermo; Anfuso, Giorgio; Cervantes, Omar; Botero Saltaren, Camilo

\begin{abstract}
This paper provides the location, scenery and litter evaluation of 35 Colombian Caribbean beaches (9 remote, 9 village, 14 urban and 3 resort). Four litter grades were found. A: excellent (5); B: good (8); C: fair (19) and D: poor (3). A Decision Value parameter (D), for scenery gave: Class I - extremely attractive/natural, $D$ > 0.85, 6 sites; Class II - attractive/natural sites, D $=0.85-0.65,2$ sites; Class III mainly natural sites, few outstanding features, $D=0.65-0.4,1$ site; Class IV - mainly unattractive sites, $\mathrm{D}=0.4$ to zero, 6 sites; Class $\mathrm{V}$ - very unattractive sites, $\mathrm{D}=<0$, 20 sites. Litter amounts placed most beaches into a poor scenic category and many scenic beaches could jump a grade by means of clean-ups. A graphic methodology highlighted beaches with contradictory results for litter/scenic grades. Tourists abhor littered beaches and clean-ups would improve scenery scores.
\end{abstract}

\section{Keywords}

Beach management, Colombia, Litter, Scenery, Tourism 